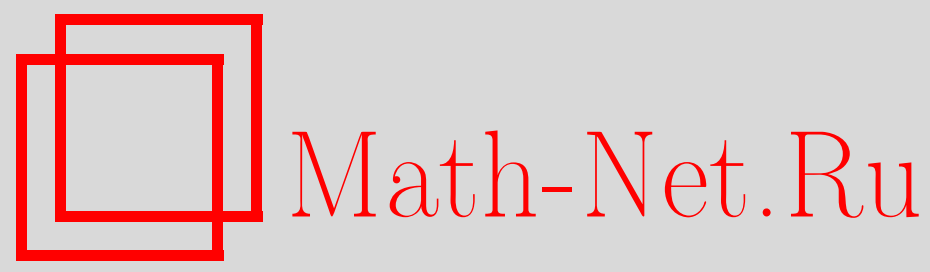

Т. И. Ишанкулов, О. И. Махмудов, Задача Коши для системы уравнений термоупругости в пространстве, Mатем. заметки, 1998, том 64, выпуск 2, 212-217

DOI: https://doi.org/10.4213/mzm1388

Использование Общероссийского математического портала Math-Net.Ru подразумевает, что вы прочитали и согласны с пользовательским соглашением http: //www . mathnet.ru/rus/agreement

Параметры загрузки:

IP: 3.93 .64 .190

26 апреля 2023 г., 15:50:35

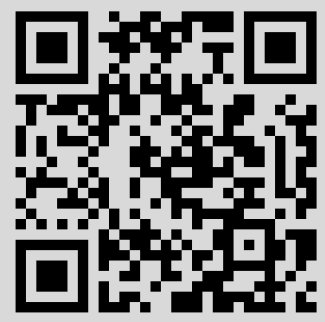




\title{
ЗАДАЧА КОШИ ДЛЯ СИСТЕМЫ УРАВНЕНИЙ ТЕРМОУПРУГОСТИ В ПРОСТРАНСТВЕ
}

\author{
Т.И. Ишанкулов, О.И. Махмудов
}

Рассматривается задача продолжения решения системы уравнений термоупругости в пространственной ограниченной области по ее значениям и значениям ее напряжений на части границы этой области, т.е. задача Коши. Строится приближенное решение этой задачи, основанное на методе функции Карлемана.

Библиография: 14 названий.

В статье рассматривается задача аналитического продолжения решения системы уравнений термоупругости в пространственной ограниченной области по ее значениям и значениям ее напряжения на части границы этой области, т.е. задача Коши. Система уравнений термоупругости эллиптическая, задача Коши для эллиптических уравнений неустойчива относительно малого изменения данных, т.е. некорректна (пример Адамара). В некорректных задачах теорема существования не доказывается, существование предполагается заданным априори. Более того, предполагается, что решение принадлежит некоторому заданному подмножеству функционального пространства, обычно компактному [1]. Единственность решения следует из общей теоремы Холмгрена [2].

На протяжении последних десятилетий не ослабевал интерес к классической некорректной задаче математической физики. Это направление в исследовании свойств решений задачи Коши для уравнения Лапласа началось в 50-х годах в работах М. М. Лаврентьева, С.Н. Мергеляна, В.К. Иванова и развивалось впоследствии В.Г. Мазьей и В.П. Хавиным, Ш. Я. Ярмухамедовым и другими [3]-[12].

Пусть $x=\left(x_{1}, x_{2}, x_{3}\right), y=\left(y_{1}, y_{2}, y_{3}\right)$ - точки трехмерного евклидова пространства $E^{3}$ и термоупругая среда есть ограниченная односвязная область $D$ в $E^{3}$ с кусочно-гладкой границей, состоящей из куска $\Sigma$ плоскости $y_{3}=0$ и гладкой поверхности $S$, лежащей в полупространстве $y_{3}>0$, т.е. $\partial D=S \cup \Sigma$. Рассмотрим матричньй дифференциальный оператор

$$
\begin{gathered}
B\left(\frac{\partial}{\partial x}, w\right)=\left\|B_{m j}\left(\frac{\partial}{\partial x}, w\right)\right\|_{4 \times 4}, \\
B_{m j}\left(\frac{\partial}{\partial x}, w\right)=\left(1-\delta_{m 4}\right)\left(1-\delta_{4 j}\right)\left(\delta_{m j} \mu\left(\Delta\left(\frac{\partial}{\partial x}\right)+\frac{\rho \omega^{2}}{\mu}\right)+(\lambda+\mu) \frac{\partial^{2}}{\partial x_{m} \partial x_{j}}\right) \\
-\delta_{4 j}\left(1-\delta_{m 4}\right) \gamma \frac{\partial}{\partial x_{m}}+i \omega \eta \delta_{m 4}\left(1-\delta_{4 j}\right) \frac{\partial}{\partial x_{j}}+\delta_{m 4} \delta_{4 j}\left(\Delta\left(\frac{\partial}{\partial x}\right)+\frac{i \omega}{\varkappa}\right), \\
m, j=1,2,3,4
\end{gathered}
$$


где

$$
\Delta\left(\frac{\partial}{\partial x}\right)=\frac{\partial^{2}}{\partial x_{1}^{2}}+\frac{\partial^{2}}{\partial x_{2}^{2}}+\frac{\partial^{2}}{\partial x_{3}^{2}}
$$

- оператор Лапласа, $\lambda, \mu$ и $\rho$ - постоянные Ламе и плотность упругой среды соответственно, $\omega$ - частота колебания, $\delta_{m j}-$ символ Кронекера, $\gamma=(3 \lambda+2 \mu) \alpha, \alpha-$ коэффициент линейного температурного расширения среды, $\varkappa$ - коэффициент температуропроводности среды, $i$ - мнимая единица, $k$ - коэффициент теплопроводности среды, $\eta=\gamma \Theta_{0} / k, \Theta_{0}$ - температура среды в недеформированном состоянии. Уравнение термоупруго-колебательного состояния среды $D$ в компонентах смешения принимает вид [13]

$$
B\left(\frac{\partial}{\partial x}, \omega\right) U=0
$$

где $U=\left(u_{1}, u_{2}, u_{3}, u_{4}\right)=\left(u, u_{4}\right), u_{1}, u_{2}, u_{3}$ - компоненты смешения, $u_{4}=\Theta$ - отклонение температуры среды от температуры $\Theta_{0}$. Решение $U$ системы $(1)$ в области $D$ назовем регулярным, если $U \in C^{1}(\bar{D}) \cap C^{2}(D)$.

В дальнейшем будем использовать матричньй дифференциальньй оператор термонапряжения

$$
R\left(\frac{\partial}{\partial y}, n(y)\right)=\left(\begin{array}{cccc} 
& & & -\gamma n_{1} \\
& T & & -\gamma n_{2} \\
& & & -\gamma n_{3} \\
0 & 0 & 0 & \frac{\partial}{\partial n}
\end{array}\right),
$$

где $T$ - оператор напряжения

$$
T\left(\frac{\partial}{\partial y}, n(y)\right)=\left\|T_{m j}\right\|_{3 \times 3}=\left\|\lambda n_{m} \frac{\partial}{\partial y_{j}}+\mu n_{j} \frac{\partial}{\partial y_{m}}+\mu \delta_{m j} \frac{\partial}{\partial n}\right\|_{3 \times 3},
$$

$n(y)=\left(n_{1}, n_{2}, n_{3}\right)$ - единичный вектор внешней нормали к поверхности $\partial D$ в точке $y$. Через $\widetilde{R}(\partial / \partial y, n(y))$ обозначим матрицу, которая получается из $R(\partial / \partial y, n(y))$ заменой в последнем столбце $\gamma$ на $i \omega \eta$.

ПоСТАНОВКА ЗАДАЧИ. Найти регулярное решение $U$ системы (1) в области $D$, исходя из ее данных Коши на поверхности $S$ :

$$
\left.U(y)\right|_{S}=f(y),\left.\quad R\left(\frac{\partial}{\partial y}, n(y)\right) U(y)\right|_{S}=g(y),
$$

$f=\left(f_{1}, f_{2}, f_{3}, f_{4}\right), g=\left(g_{1}, g_{2}, g_{3}, g_{4}\right)$ - заданные непрерывные вектор-функции.

Данная задача является некорректной. Характер некорректности такой же, как у задачи Коши для уравнения Гельмгольца. Для случая $\omega=0, \gamma=0$ [7] (система уравнений статики) рассматриваемая задача исследована в [11]. Наша цель состоит в построении приближенного решения задачи (1), (2), основанном на методе функции Карлемана.

Функцию Карлемана задачи Коши для уравнения Лапласа и ему близких в случае, когда $\Sigma$ - часть поверхности конуса, построил Ярмухамедов [6], [7]. Матрицу Карлемана задачи Коши для уравнений Коши-Римана в случае, когда $S$ - произвольное множество положительной меры, построил Л.А. Айзенберг [9]. Развивая идею Мергеляна [5], указавшего (основывающийся на теоремах об аппроксимации) способ построения функции 
Карлемана задачи Коши для уравнения Лапласа в случае, когда $S$ - кусок гранищы односвязной области с гладким краем, Н. Н. Тарханов [12] построил матрицу Карлемана для эллиптических систем.

Пусть вместо $f(y)$ и $g(y)$ заданы с точностью $\delta, 0<\delta<1$ (в метрике $C$ ), их приближения $f_{\delta}(y)$ и $g_{\delta}(y)$, которые могут не принадлежать классу существования решений. Построим семейство функций $U\left(x, f_{\delta}, g_{\delta}\right)=U_{\sigma \delta}(x)$, зависящее от параметра $\sigma$, и докажем, что при некоторых условиях и специальном выборе параметра $\sigma(\delta)$ при $\delta \rightarrow 0$ семейство $U_{\sigma \delta}(x)$ сходится в обычном смысле к решению $U(x)$ задачи $(1),(2)$. Следуя А. Н. Тихонову [14], назовем $U_{\sigma \delta}(x)$ регуляризованным решением задачи. Регуляризованное решение определяет устойчивьй метод приближенного решения задачи $(1),(2)$.

Используя результаты Лаврентьева и Ярмухамедова [1], [4], [6] относительно задачи Коши для уравнения Лапласа, нам удалось построить матрицу Карлеманав явном виде и на ее основе регуляризованное решение задачи Коши для систем уравнений термоупругости.

Ранее в работах [9], [12] было доказано, что матрица Карлемана существует во всякой задаче Коши для эллиптических систем, если только данные Коши задаются на граничном множестве с положительной мерой. Поскольку речь идет о явных формулах, построение матрицы Карлемана в элементарных и специальных функциях представляет значительньй интерес.

Следуя [1], приведем

ОПРЕДЕЛЕНИЕ. Матриией Карлемана задачи $(1),(2)$ назьвается $(4 \times 4)$-матрица $\Pi(y, x, \omega, \gamma, \sigma)$, удовлетворяющая следующим двум условиям:

$$
\Pi(y, x, \omega, \gamma, \sigma)=\Gamma(y, x, \omega, \gamma)+G(y, x, \omega, \gamma, \sigma)
$$

где $\sigma$ - положительньй числовой параметр, матрица $G(y, x, \omega, \gamma, \sigma)$ по переменной $y$ удовлетворяет системе $(1)$ всюду в области $D, \Gamma(y, x, \omega, \gamma)$ - матрица фундаментальных решений уравнения (1);

$$
\int_{\Sigma}\left(|\Pi|+\left|\left(\widetilde{R} \Pi^{*}\right)^{*}\right|\right) d s_{y} \leqslant \varepsilon(\sigma),
$$

где $\varepsilon(\sigma) \rightarrow 0$ при $\sigma \rightarrow \infty$; здесь и далее $\Pi^{*}$ - матрица сопряженная $\Pi$, а $|\Pi|$ - евклидова норма матрицы $\Pi=\left\|\Pi_{m j}\right\|$, т.е. $|\Pi|=\left(\sum_{m, j=1}^{4} \Pi_{m j}^{2}\right)^{1 / 2}$. В частности, для вектора $U=\left(u, u_{4}\right)$ имеем $|U|=\left(\sum_{m=1}^{4} u_{m}^{2}\right)^{1 / 2}$.

С целью построения приближенного решения задачи $(1),(2)$ рассмотрим матрищу

$$
\begin{aligned}
& \Pi(y, x, \omega, \gamma, \sigma)=\left\|\Pi_{m j}(y, x, \omega, \gamma, \sigma)\right\|_{4 \times 4}, \\
& \Pi_{m j}(y, x, \omega, \gamma, \sigma) \\
& =\frac{\left(1-\delta_{m 4}\right)\left(1-\delta_{j 4}\right)}{2 \pi}\left(\frac{\delta_{m j}}{\mu} \Phi_{\sigma}\left(y, x, i \lambda_{3}\right)-\sum_{k=1}^{3} \alpha_{k} \frac{\partial^{2}}{\partial x_{m} \partial x_{j}} \Phi_{\sigma}\left(y, x, i \lambda_{k}\right)\right) \\
& +\frac{i \omega \eta}{2 \pi} \delta_{m 4}\left(1-\delta_{j 4}\right) \sum_{k=1}^{3} \beta_{k} \frac{\partial}{\partial x_{j}} \Phi_{\sigma}\left(y, x, i \lambda_{k}\right) \\
& -\frac{\gamma}{2 \pi} \delta_{j 4}\left(1-\delta_{m 4}\right) \sum_{k=1}^{3} \beta_{k} \frac{\partial}{\partial x_{m}} \Phi_{\sigma}\left(y, x, i \lambda_{k}\right)+\frac{\delta_{j 4} \delta_{m 4}}{2 \pi} \sum_{k=1}^{3} \gamma_{k} \Phi_{\sigma}\left(y, x, i \lambda_{k}\right),
\end{aligned}
$$


где постоянные $\alpha_{k}, \beta_{k}, \gamma_{k}, \lambda_{k}$ явно выражаются через постоянные термоупругости - коэффициенты системы (1) (см. [13]):

$$
\begin{gathered}
\Phi_{\sigma}(y, x, \Lambda)=\frac{1}{2 \pi} \int_{0}^{\infty} \operatorname{Im} \frac{\exp \left(\sigma\left(W-x_{3}\right)+\left(W-x_{3}\right)^{2}\right)}{W-x_{3}} \frac{\cos \Lambda u d u}{\sqrt{u^{2}+\alpha^{2}}}, \\
W=i \sqrt{u^{2}+\alpha^{2}}+y_{3}, \quad \alpha^{2}=\left(y_{1}-x_{1}\right)^{2}+\left(y_{2}-x_{2}\right)^{2}, \quad \alpha>0 .
\end{gathered}
$$

Из результатов работы [6] вытекает

ЛЕмма 1. Функиия $\Phi_{\sigma}(y, x, \Lambda)$ является функиией Карлемана для уравнения Гельмгольиа, т.е. обладает следующими двумя свойствами:

$$
\Phi_{\sigma}\left(y, x, i \Lambda_{k}\right)=\frac{\exp (\Lambda r)}{4 \pi r}+v(y, x, \Lambda, \sigma), \quad r=|x-y|,
$$

где $v(y, x, \Lambda, \sigma)$ - некоторая функиия, определенная для всех значений $y, x$ и удовлетворяюшая уравнению Гельмгольиа

$$
\Delta\left(\frac{\partial}{\partial y}\right) v-\Lambda^{2} v=0, \quad y \in D, \quad \int_{\Sigma}\left(\left|\Phi_{\sigma}\right|+\left|\frac{\partial \Phi_{\sigma}}{\partial n}\right|\right) d s_{y} \leqslant C(\Lambda, D) \sigma \exp \left(-\sigma x_{3}\right)
$$

әде $C(\Lambda, D)$ - постоянная.

Докажем аналогичную лемму для системы (1).

Лемма 2. Матрица $\Pi(y, x, \omega, \gamma, \sigma)$, определенная равенствами (3) $и$ (4), является матрицей Карлемана для задачи (1), (2).

ДокАЗАТЕЛЬСтво. В силу равенств (3) и (5) имеем

$$
\begin{aligned}
& \Pi(y, x, \omega, \gamma, \sigma)=\Gamma(y, x, \omega, \gamma)+\| \frac{\left(1-\delta_{m 4}\right)\left(1-\delta_{j 4}\right)}{2 \pi}\left(\frac{\delta_{m j}}{\mu} v\left(y, x, i \lambda_{3}, \sigma\right)\right. \\
& \left.\quad-\sum_{k=1}^{3} \alpha_{k} \frac{\partial^{2}}{\partial x_{m} \partial x_{j}} v\left(y, x, i \lambda_{k}, \sigma\right)\right)+\frac{i \omega \eta}{2 \pi} \delta_{m 4}\left(1-\delta_{m 4}\right) \sum_{k=1}^{3} \beta_{k} \frac{\partial}{\partial x_{j}} v\left(y, x, i \lambda_{k}, \sigma\right) \\
& \quad-\frac{\gamma}{2 \pi} \delta_{j 4}\left(1-\delta_{m 4}\right) \sum_{k=1}^{3} \beta_{k} \frac{\partial}{\partial x_{m}} v\left(y, x, i \lambda_{k}, \sigma\right)-\frac{\delta_{j 4} \delta_{m 4}}{2 \pi} \sum_{k=1}^{3} \gamma_{k} v\left(y, x, i \lambda_{k}, \sigma\right) \| \\
& =\Gamma(y, x, \omega, \gamma)+\left\|G_{m j}(y, x, \omega, \gamma, \sigma)\right\|=\Gamma(y, x, \omega, \gamma)+G(y, x, \omega, \gamma, \sigma) .
\end{aligned}
$$

Непосредственным вычислением можно убедиться, что матрица $G(y, x, \omega, \gamma, \sigma)$ по переменному $y$ удовлетворяет системе $(1)$ всюду в области $D$.

Используя формулы $(3),(4)$ и (6) на каждом компакте $D^{\prime} \subset D$, получим

$$
\int_{\Sigma}\left(|\Pi|+\left|\widetilde{R} \Pi^{*}\right|\right) d s \leqslant C(\lambda, \mu, \omega, \gamma, D) \sigma^{2} \exp \left(-\sigma x_{3}\right),
$$

где $C(\lambda, \mu, \omega, \gamma, D)$ - некоторая постоянная. Здесь и далее, для краткости обозначений опускаем аргументы функции П. Лемма доказана.

Положим

$$
2 U_{\sigma}(x)=\int_{S}\left(\Pi R U(y)-\left(\widetilde{R} \Pi^{*}\right)^{*} U(y)\right) d s_{y}, \quad x \in D
$$

Имеет место 
ТЕОрема 1. Пусть $U(x)$ - регулярное решение системы (1) в области D, удовлетворяющее условию

$$
|U(x)|+\left|R\left(\frac{\partial}{\partial y}, n\right) U(x)\right| \leqslant 1, \quad y \in \Sigma .
$$

Тогда при $\sigma \geqslant 1$ справедлива оченка

$$
\left|U(x)-U_{\sigma}(x)\right| \leqslant C(\lambda, \mu, \omega, \gamma, D) \sigma^{2} \exp \left(-\sigma x_{3}\right), \quad x \in D
$$

ДокАЗАТЕЛЬСтво. Из леммы 2 для регулярного решения системы $(1)$ в области $D$ вытекает справедливость интегральной формулы Грина-Купрадзе [13]

$$
2 U(x)=\int_{\partial D}\left(\Pi R\left(\frac{\partial}{\partial y}, n\right) U(y)-\left(\widetilde{R}\left(\frac{\partial}{\partial y}, n\right) \Pi^{*}\right)^{*} U(y)\right) d s_{y}, \quad x \in D
$$

Перепишем это равенство в виде

$$
2 U(x)=\int_{S}\left(\Pi R U(y)-\left(\widetilde{R} \Pi^{*}\right)^{*} U(y)\right) d s_{y}+\int_{\Sigma}\left(\Pi R U(y)-\left(\widetilde{R} \Pi^{*}\right)^{*} U(y)\right) d s_{y}
$$

Неравенство (10) следует из (7) и (11). Теорема доказана.

Приведем результат, которьй позволяет вычислить $U(x)$ приближенно, когда на поверхности $S$ вместо $U(x)$ и $R(\partial / \partial y, n(y)) U(x)$ заданы их непрерьвные приближения $f_{\delta}(y)$ и $g_{\delta}(y)$ :

$$
\max _{S}\left|f(y)-f_{\delta}(y)\right|+\max _{S}\left|g(y)-g_{\delta}(y)\right| \leqslant \delta, \quad 0<\delta<1
$$

Предположим, что $S$ удовлетворяет условиям Ляпунова. Функцию $U_{\sigma \delta}(x)$ определим формулой

$$
\begin{gathered}
2 U_{\sigma \delta}(x)=\int_{S}\left(\Pi g_{\delta}(y)-\left(R\left(\frac{\partial}{\partial y}, n\right) \Pi^{*}\right)^{*} f_{\delta}(y)\right) d s_{y}, \quad x \in D \\
\sigma=\frac{1}{x_{3}^{0}} \ln \frac{1}{\delta}, \quad x_{3}^{0}=\max _{D} x_{3}
\end{gathered}
$$

ТеОрема 2. Пусть $U(x)$ - регулярное решение системы (1) в области D, удовлетворяющее условию (9). Тогда справедлива оценка

$$
\left|U(x)-U_{\sigma \delta}(x)\right| \leqslant C(\lambda, \mu, \omega, \gamma, D) \delta^{x_{3} / x_{3}^{0}}\left(\ln \frac{1}{\delta}\right)^{3}, \quad x \in D^{\prime} \subset D
$$


ДоКАЗАТЕЛЬСТво. Из формул (8), (11) и (13) для любого $x \in D$ вытекает

$$
\begin{aligned}
\mid U(x) & -U_{\sigma \delta}(x) \mid \\
= & \frac{1}{2} \int_{S}\left(\Pi\left(R\left(\frac{\partial}{\partial y}, n\right) U(y)-g_{\delta}(y)\right)-\left(\widetilde{R}\left(\frac{\partial}{\partial y}, n\right) \Pi^{*}\right)^{*}\left(U(y)-f_{\delta}(y)\right)\right) d s_{y} \\
& +\int_{\Sigma}\left(\Pi R\left(\frac{\partial}{\partial y}, n\right) U(y)-\left(\widetilde{R}\left(\frac{\partial}{\partial y}, n\right) \Pi^{*}\right)^{*} U(y)\right) d s_{y}, \quad x \in D .
\end{aligned}
$$

Из условия теоремы и неравенств $(7),(10),(12)$ имеем

$$
\begin{aligned}
\left|U(x)-U_{\sigma \delta}(x)\right| & \leqslant C_{1}(\lambda, \mu, \omega, \gamma, D) \sigma^{3} \exp \left(\sigma x_{3}^{0}-\sigma x_{3}\right)+C(\lambda, \mu, \omega, \gamma, D) \sigma^{3} \exp \left(-\sigma x_{3}\right) \\
& \leqslant C_{2}(\lambda, \mu, \omega, \gamma, D) \sigma^{3}\left(1+\delta \exp \left(\sigma x_{3}^{0}\right)\right) \exp \left(-\sigma x_{3}\right), \quad x \in D .
\end{aligned}
$$

Согласно выбору (14) параметра $\sigma$ из (15) следует утверждение теоремы.

СлЕдСТВИЕ. Предельные равенства

$$
\lim _{\sigma \rightarrow \infty} U_{\sigma}(x)=U(x), \quad \lim _{\delta \rightarrow \infty} U_{\sigma \delta}(x)=U(x)
$$

выполняются равномерно на каждом компакте из D. Формула (8) дает в явном виде приближенное решение задачи (1), (2), а формула (13) - приближснное решение, когда данные Коши на $S$ заданы приближснно.

В заключение авторы выражают признательность академику М. М. Лаврентьеву и профессору Ш.Я. Ярмухамедову за постановку задачи и обсуждения в процессе ее решения.

\section{СПИСОК ЦИТИРОВАННОЙ ЛИТЕРАТУРЫ}

[1] Лаврентьев М. М. О некоторых некорректных задачах математической физики. Новосибирск: ВЦ СО АН СССР, 1962.

[2] Петровский И. Г. Лекции об уравнениях с частными производными. М.: Физматгиз, 1961.

[3] Лаврентьев М. М. О задаче Коши для уравнения Лапласа // Изв. АН СССР. Сер. матем. 1956. T. 20. №6. С. $819-842$.

[4] Лаврентьев М. М. О задаче Коши для линейных эллиптических уравнений второго порядка // Докл. АН СССР. 1957. Т. 112. № 2. С. 195-197.

[5] Мергелян С. Н. Гармоническая аппроксимация и приближенное решение задачи Коши для уравнения Лапласа // УМН. 1956. Т. 11. № 5. С. 3-26.

[6] Ярмухамедов Ш.Я. О задаче Коши для уравнения Лапласа // Докл. АН СССР. 1977. Т. 235 . № 2. С. 281-283.

[7] Ярмухамедов Ш. Я., Ишанкулов Т. И., Махмудов О. И. О задаче Коши для системы уравнений теории упругости в пространстве // Сиб. матем. ж. 1992. Т. 33. № 1. С. 186-190.

[8] Иванов В. К. Задача Коши для уравнения Лапласа в бесконечной полосе // Дифференц. уравнения. 1965. Т. 1. С. 131-136.

[9] Айзенберг Л.А., Тарханов Н.Н. Абстрактная формула Карлемана // Докл. AH CCCP. 1988. Т. 298. №6. С. 1292-1296.

[10] Махмудов О. И. Задача Коши для системы теории упругости в пространстве. Дисс. ... к. ф.-м. н. Новосибирск, 1990.

[11] Махмудов О. И. Задача Коши для системы уравнений пространственной теории упругости в перемещениях // Изв. вузов. Матем. 1994. № 1. С. 54-61.

[12] Тарханов Н. Н. О матрице Карлемана для эллиптических систем // Докл. АН СССР. 1985. T. 284. № 2. C. 294-297.

[13] Купрадзе В. Д., Гегелиа Т. Г., Башелейшвили М. О., Бурчуладзе Т. В. Трехмерные задачи математической теории упругости и термоупругости. М.: Наука, 1976.

[14] Тихонов А.Н. О решении некорректно поставленных задач и методе регуляризации // Докл. АН СССР. 1963. Т. 151. № 3. С. 501-504. 